\title{
COVID-19 DATA COMPARISON OF WORLD, INDIA AND TAMIL NADU BY SIR MODEL WITH DIFFERENT TRANSMISSION AND RECOVERY COEFFICIENT
}

\author{
B. VENNILA \& J. SASIKUMAR
}

Department of Mathematics, School of Basic Sciences, College of Engineering and Technology, SRM Institute of Science and

Technology, Kattankulathur, Tamil Nadu, India

\begin{abstract}
The interest in writing this paper is to know the endemic stage of COVID19 by using the SIR mathematical model. For this purpose confirmed, recovered data have been collected from statista.com on April 1.The original population is shared with the above data to predict the future control of COVID- 19 by the SIR model. Results are graphically represented for 60 days starting from April 1 to May 31 with varying the parameters and the table values inserted in Google spreadsheet and the link is given last. In this model transmission and recovery coefficient playing an important role. This model was implemented using Python.

KEYWORDS: Transmission Coefficient, Recovery Coefficient ,Population, Statistical Data
\end{abstract}

Received: Jun 08, 2020; Accepted: Jun 28, 2020; Published: Aug 11, 2020; Paper Id.: IJMPERDJUN2020732

\section{INTRODUCTION}

COVID19 is a highly transmittable respiratory disease which is caused by SARS corona virus. The serious virus emerged in china December 2019 and rapidly increases to all over world, till persisting everywhere. Corona virus are a huge people of viruses that are recognized to origin illness ranging from the ordinary freezing to more cruel diseases such as Middle East Respiratory Syndrome (MERS) and Severe Acute Respiratory Syndrome (SARS). A fresh corona virus that has not been formally recognized in humans. In a meeting on January 30, 2020, the outbreak was declared by the WHO, the virus could also be transmitted from human to human and symptomatic people are the most repeated source of COVID19 extend. This data suggests that the exercise of quarantine is the best way of controlling this pandemic.

Preventive actions are the present approach to limit the spread of cases. Because an outbreak will boost on condition that the recovered value is bigger than one. Protective Strategies are focused on the quarantine of patients and careful contamination control. The occasion on or after coverage to onset of symptoms is classically in the region of two to 14 days. Suggested procedures to foil infectivity consist of regular hand washing, maintain bodily detachment commencing others (mainly from persons by manifestations), casing coughs plus sneezes through a handkerchief and keeping dirty hands departed from the face. Currently, there is no vaccine treatment forCOVID19.

Davil.s.Hui et al[1] analyzed the ongoing covid -19plaguerisk of original corona viruses to worldwide health- The hottest 2019 novel corona virus outburst I Wuhan, China. Wujt et al[2] studied the Now casting and forecasting the possible marital and global multiply of the 2019-nCoV outreaching in Wuhan; a modeling study. liQ et al[3] analyzed figures on the first 425 established cases in Wuhan to find out the epidemiologic personality of NICP. Benjamin et al[4] developed an O- SEIHRD exact model for the stretch of the corona virus disease(COVID19). Qianyinglin et al[5] discussed the individual reaction and governmental action during the COVID-19 outbreak 
in Wuhan. Prem et al [6] examined how changes in population mixing have affected outbreak progression in Wuhan.

Cleo Anastassopulou et al[7] calculated data-based scrutiny, modeling, and forecasting of the COVID-19 occurrence. Fathalla et al [8] investigated the subjective behavior of the replica and discover the situation that security the asymptotic firmness of consequent fixed states. BinitiHamzahet al [9] used to deduce patterns of civic attitude of disseminating linked health information and evaluate the supporting and monetary influence of the spread of the virus. Weston et al [10] established that non- identifiability in model calibrations by means of the confirmed-case data is the foremost reason for such extensive variations. Yu [11] simplified estimating the tainted residents of Wuhan corona virus in special policy scenarios by the SIR model

Zhihua Bai et al[13] studied the hastyre view as well as premature warnings are essential for outbreak investigation in response to grave public health proceedings. Benjamin et al[14] analyzed the recent outbreak of COVID19 in mainland China was characterized by aidiosyncraticsub exponential augment of inveterate cases during the early phase of the epidemic contrasting an exponential growth expected for an unimpeded outbreak. Steven Sanche et al[15] composed general entity case news across China and predictable key epidemiologic parameters, including the incubation period.

David Smith et al [16] identified the independent and dependent variables for the SIR modeling process. Ottar et al [17] developed a TSIR model that captures both endemic cycles and episodic outbreak in measles. Jiajia et al [18] introduced the two groups of SIR models for allowing random fluctuation around the endemic equilibrium.

\section{METHODOLOGY}

Basic hypothesis of the SIR model

As the first pace in the modeling route, the free and reliant variables are to be known The independent variable is time $t$, considered in days.

We consider two set of dependent variables counts people in each of the groups, each as a function of time

Susceptible class, $S=S(t)=$ figure of susceptible - individuals who may catch the disease but at present are not infected

Infective class, $I=I(t)=$ figure of infective - individuals who are infected with the disease and currently transmittable

Removed class, $R=R(t)=$ figure of removals - those who cannot get the disease because they have improved permanently, or obviously immune or have died.

The next set of dependent variables represents the division of the entire population in each of the three categories. So, if $\mathrm{N}$ is the total population, we have 


$$
\begin{aligned}
& s(t)=\frac{S(t)}{N} \text {, the susceptible portion of the population, } \\
& i(t)=\frac{I(t)}{N} \text { the infected part of the population, } \\
& r(t)=\frac{R(t)}{N} \text {, the recovered small part of the population } \\
& \text { Each time } s(t)+i(t)+r(t)=1 \\
& \frac{d s}{d t}=-a s(t) i(t) \\
& \frac{d i}{d t}=-a s(t) i(t)-b i(t) \\
& \frac{d r}{=}=b i(t) d t
\end{aligned}
$$

We have considered the initial population variables as of January 2020 and Susceptible, Infectious, recovered values on April 1 in the world, India, and Tamil Nadu

$$
\begin{aligned}
& \mathrm{S}(0)=7763035303 \\
& \mathrm{I}(0)=932475 \\
& \mathrm{R}(0)=240360 \\
& \mathrm{~s}(0)=0.9998511 \\
& \mathrm{i}(0)=0.0001184 \\
& \mathrm{r}(0)=0.0000305 \\
& \mathrm{~S}(0)=1387297452 \\
& \mathrm{I}(0)=1998 \\
& \mathrm{R}(0)=206 \\
& \mathrm{~s}(0)=0.999998412 \\
& \mathrm{i}(0)=0.00000144 \\
& \mathrm{r}(0)=0.00000148 \\
& \mathrm{~S}(0)=0.999996877
\end{aligned}
$$


$\mathrm{i}(0)=0.000003032$

$r(0)=0.000000091$

\section{PYTHON CODE}
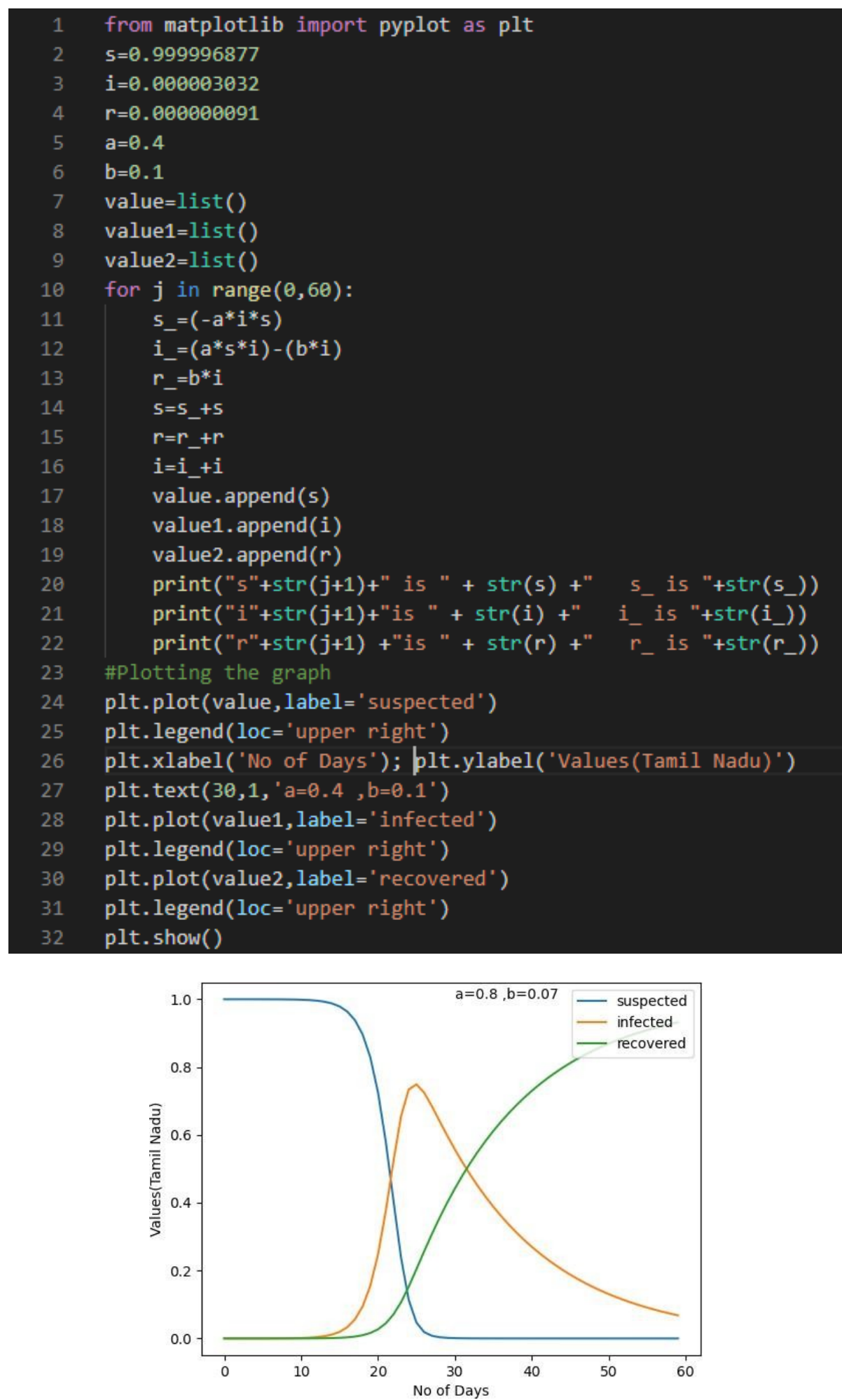

Figure 1: Tamil Nadu Data of COVID-19 with Transmission Coefficient ' $a$ ' $=0.8$ and Recovered Coefficient ' $b$ ' $=0.07$. 


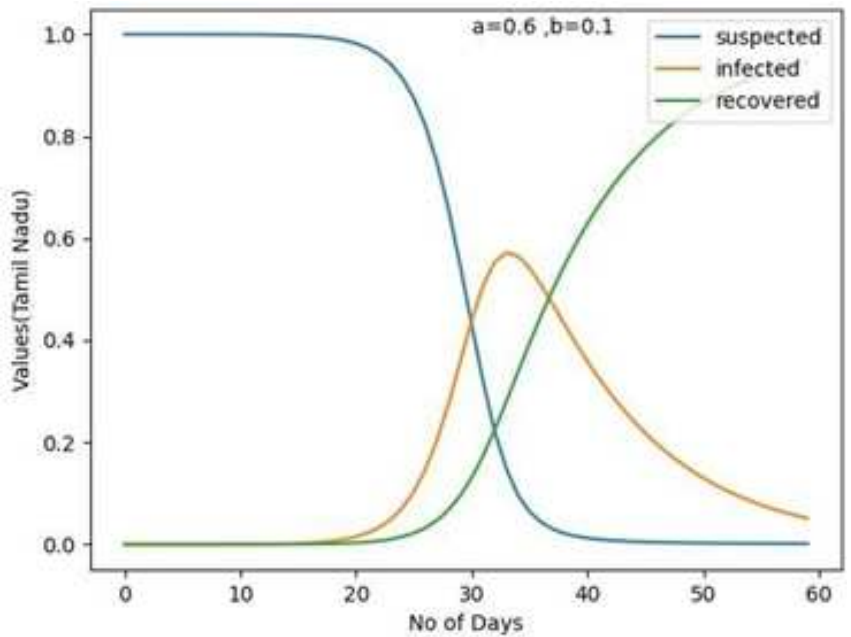

Figure 2: Tamil Nadu Data of COVID-19 with Transmission Coefficient ' $a$ ' $=0.6$ and Recovered Coefficient ' $b$ ' $=0.1$.

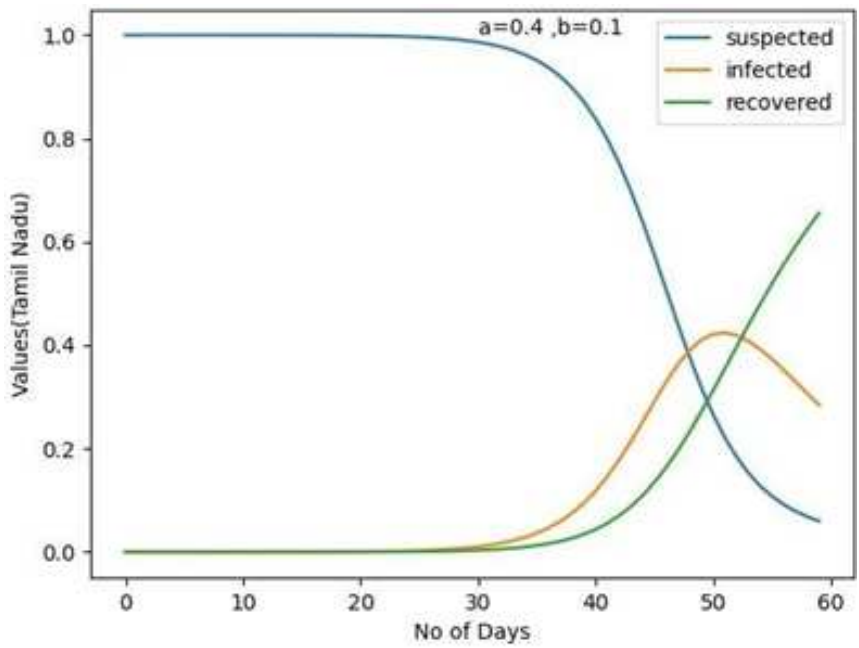

Figure 3: Tamil Nadu Data of COVID-19 with Transmission Coefficient ' $a$ ' $=0.6$ and Recovered Coefficient ' $b$ ' $=0.1$.

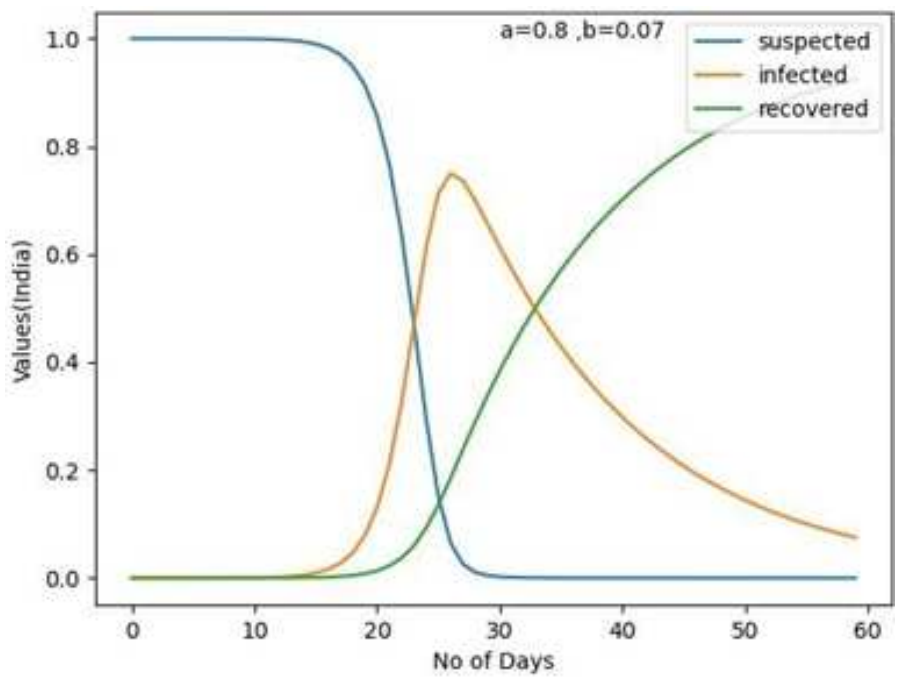

Figure 4: India Data of COVID-19 with Transmission Coefficient ' $a$ ' $=0.8$ and Recovered Coefficient ' $b$ ' $=0.07$. 


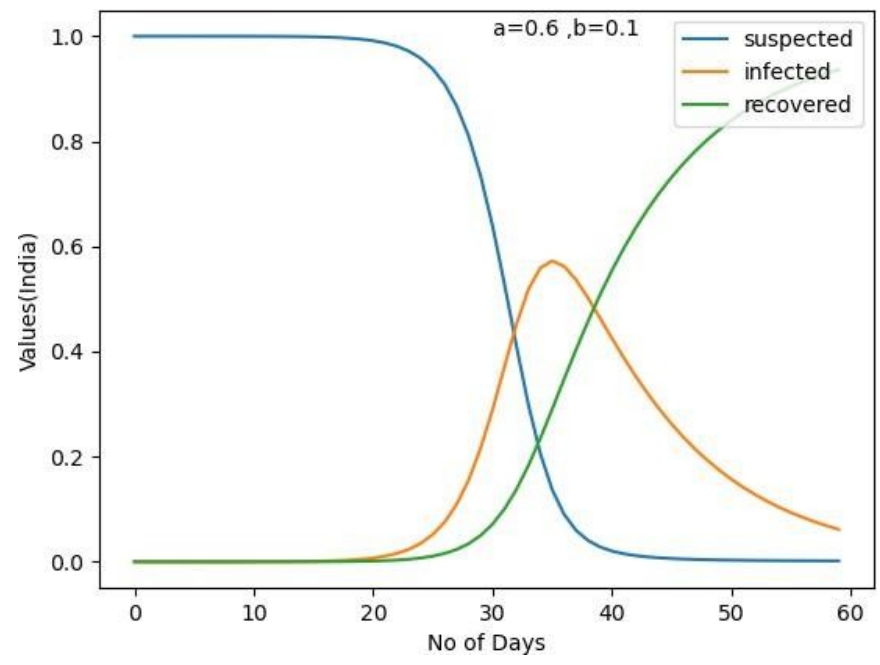

Figure 5: India Data of COVID-19 with Transmission Coefficient ' $a$ ' $=0.6$ and Recovered Coefficient ' $b$ ' $=0.1$.

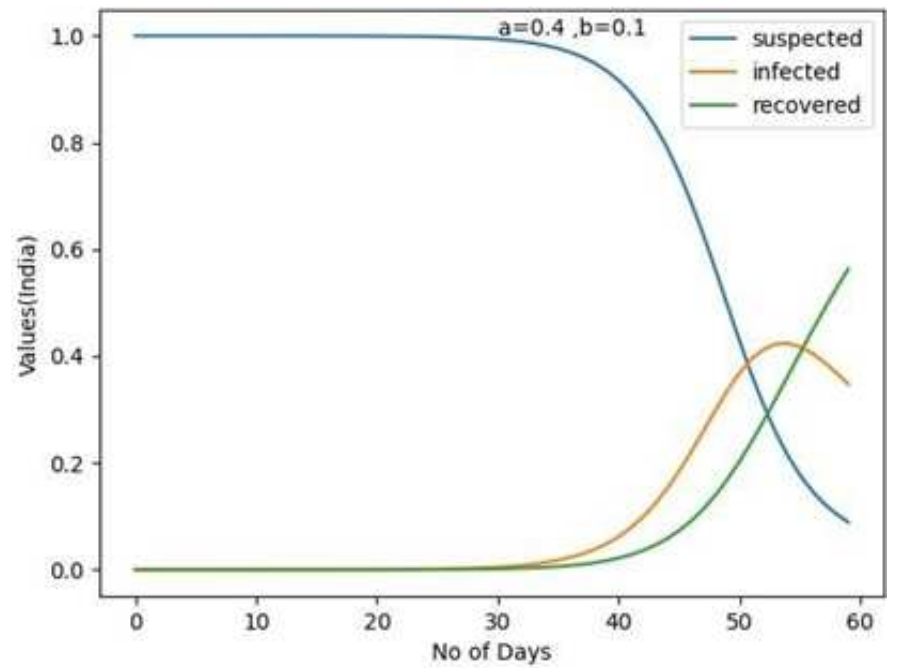

Figure 6: India Data of COVID-19 with Transmission Coefficient ' $a$ ' $=0.4$ and Recovered Coefficient ' $b$ ' $=0.1$.

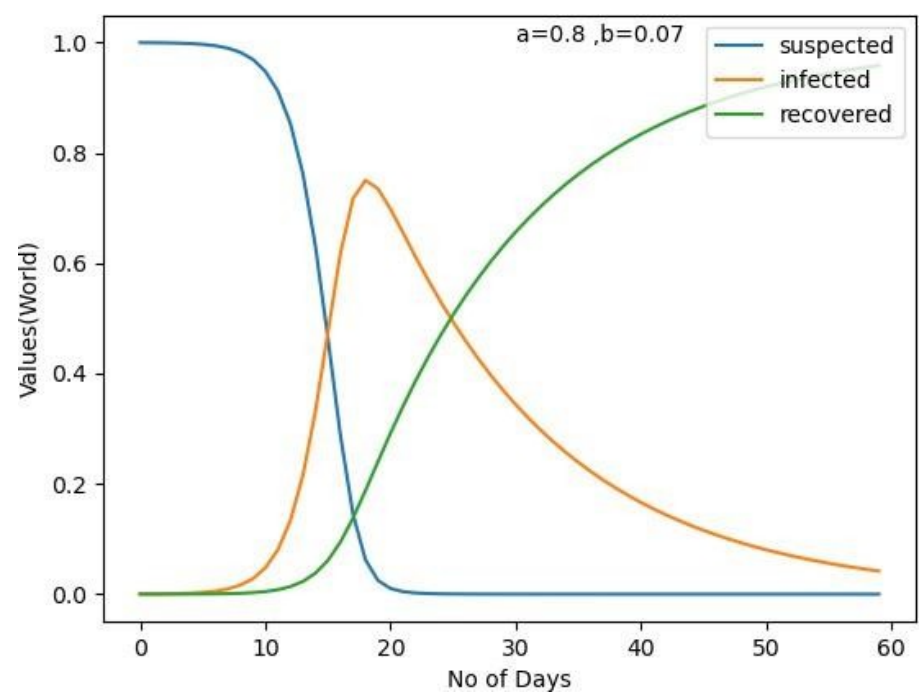

Figure 7: World Data of COVID-19 with Transmission Coefficient ' $a$ ' $=0.4$ and Recovered Coefficient ' $b$ ' $=0.1$. 


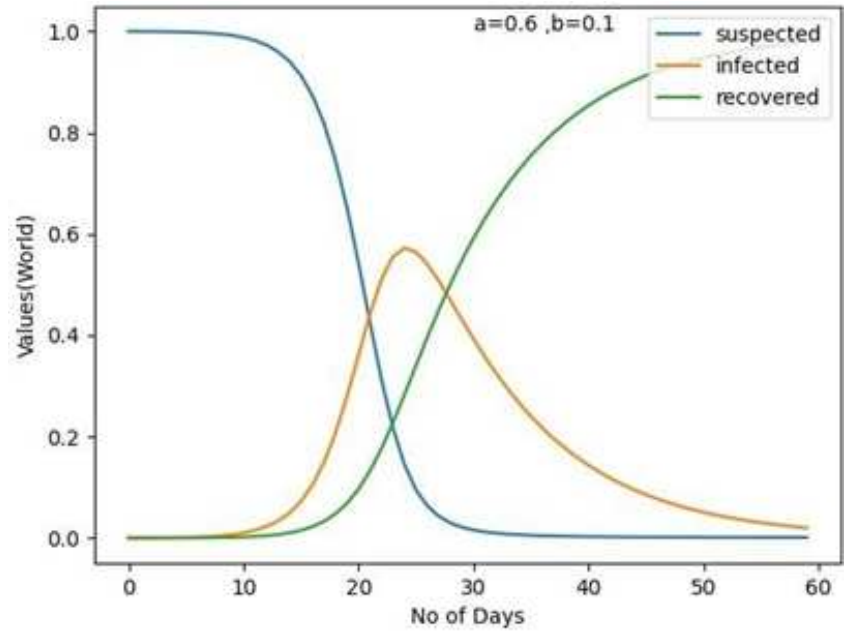

Figure 8: World Data of COVID-19 with Transmission Coefficient ' $a$ ' $=0.6$ and Recovered Coefficient ' $b$ ' $=0.1$.

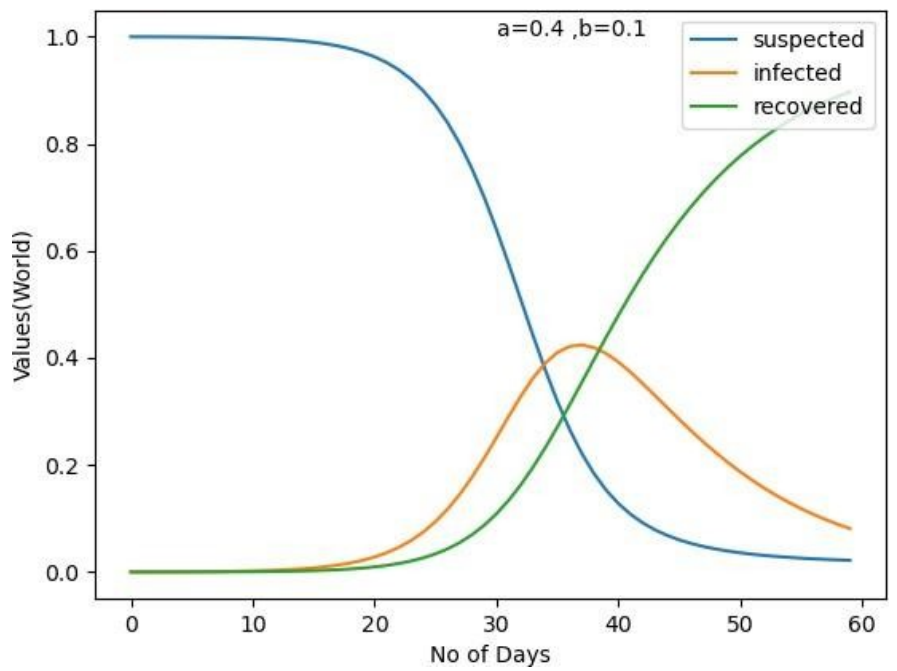

Figure 9: World Data of COVID-19 with Transmission Coefficient ' $a$ ' $=0.4$ and Recovered Coefficient ' $b$ ' $=0.1$.

\section{RESULTS AND DISCUSSION}

All the Figures have been generated from April 1 to May 31, 2020 totally 0 to 60 days. In figure 1, 4 and 7 it is seen that infected is peak on April 20 for world, April 27 for Tamil Nadu and April 29 for India. After this gradually decreasing trend is shown in the figure. But suspected is high on April 8 for world, April 16 for Tamil Nadu and April 18 for India, after this decrease trend is appearing in the figures. Coming to the recovering stage after April 14 for World, April 21 for Tamil Nadu, April 23 for India. After that increasing trend is exposed .From figures 2, 5, 8, it is clear that the suspected level is high on April 12 for World, on April 22 for Tamil Nadu, and on April 20 for India. Infected level is identified high for World on April 26, Tamil Nadu on May 5 and May 7 for India. Recovering is gradually increasing from April 18 for World, April 27 for Tamil Nadu and April 29 for India. From figures 3, 6 and 8,it is noted that suspected level is top on April 18 for world, May 1for Tamil Nadu and May 2 for India. Infected level is high on May 9 for world, May 23 for Tamil Nadu and May 26 for India. Based on Infected level recovering getting increasing trend. 


\section{CONCLUSIONS}

Based on statistics provided with transmission coefficient ' $a$ ' $=0.8$ and recovery coefficient ' $b$ ' $=0.07$ We are concluding that coronavirus epidemic stage will come to control stage by the middle of May for India and Tamil Nadu (16, 14), But for the World it will be by the end of this May. The above predictions are derived when certain factors influenced such as lockdown and few days of curfew, if those factors are not considered the predictions may vary accordingly. To bring this spreading of coronavirus to an end we must spend 14 days in quarantine even if we feel perfectly fine. Why 14 days exactly, a host which takes some particular time to replicate itself and then that mass begins to shack the virus from first to last coughs, sneezes for instance. This is known as the virus incubation period which varies from virus to virus and sometimes even from host to host.

\section{REFERENCES}

1. D.Hui.The continuing 2019-Ncov epidemic threat of novel coronavirus to global health. The latest 2019 novel coronavirus outbreak in Wuhan, China, International journal of Infected Disseases,2020,91,pp.264266.https://dx.doi.org/10.1016\%2Fj.ijid.2020.01.009

2. Devaraj, Manimozhi. "Inter-Intra Frame Coding in Motion Picture Compensation Using New Wavelet Bi-Orthogonal Coefficients. "International Journal of Electronics and Communication Engineering (IJECE) 5.3, Mar - Apr 2016, 1-10

3. J.J.Wu.K.Leung,G.M.Leung.Nowcasting and forecasting the potential domestic and international sread of the 2019-Ncov outbreak originating in Wuhan, China: a modeling study, The Lancet, 2020,395395,pp.689-697. doi: 10.1016/S01406736(20)30260-9. Epub 2020 Jan31.

4. LiQ,Guan.X.Wu.P.Wang.x.Zhou.Early Transmission dynamics in Wuhan, China, of Novel Coronavirus-Infected Pneumonia.N.Engg.J.Med,2020. doi: 10.1056/NEJMoa2001316. Epub 2020 Jan 29.

5. Benjamin Ivorra, MirianFerrandez Mario Velo.A.M.Ramos .Mathematical modeling of the spread Of the coronavirus diseases 2019(COVID19) taking in to account the undetected infections. The case China, Non linear Science and Numerical Simulation, 2020 doi;10.13140/RG.2.2.21543.29604

6. Isaac, Ann Maria, and K. Rathi. "Performance Analysis of Hybrid HVDC Transmission Systems for Connecting Offshore Wind Farm to Onshore Grid." International Journal of Innovative Reseach in Science, Engineering and Technology 3.5 (2014): 201-207.

7. QianyingLin,ShiZhao,DaaozhouGao,WelmingWang,LinYang,DalhalHe.A conceptual model for the coronavrus disease 2019 outbreak in wuhan, China with individual reaction and governmental action, International Journal of Infective Diseases,2020,93,pp211-216.doi;https://doi.org/10.1016/j.ijid.2020.02.058

8. K.Prem,Y.Liu,T.W.RusselT.W.Kucharaskia,r.M.Eggo,N.Davies,The effect of control straregies to reduce social mixing on outcomes of the COVID-19 epidemic in Wuhan, China, a modeling study, The lancet public health 2020 . doi:10.1016/S24682667(20)30073-6

9. Hassan, Qusay MA, et al. "Evolution of far-field diffraction patterns and nonlinear optical properties of SAE 70 oil." Int. J. Appl. Nat. Sci 6 (2017): 181-8.

10. Celo Anastassopoulou, Lucia Russo Athanasios Tsakris, Constantinossiettos,PLOS,2020,15P. doi: 10.1371/journal.pone.0230405

11. Fathalla. Rihan, M.Naim Anwar, Qualitative analysis of delayed SIR epidemic Model with saturated Incidence rate, International Journal of Differential Equations 2012.pp1-13 https://doi.org/10.1155/2012/408637 
12. F.A.BintiHamzah, C.Lau, H.Zazri, D.V, Ligot, G.Lee, C.L.Tan.Corona Tracker: World-wide COVID-19 outbreak Data Analysis and Prediction,2020,Bullitin of world Health Organizationhttp://dx.doi.org/10.2471/BLT.20.255695

13. C.Weston, Roda,B. Marie.B.Varughese, Y.Li.Michaael. Infectious Disease Modelling "why is it difficult to accurately predict the COVID-19 epidemic, Elsevier public health emergency collection,2020,5,pp 271-281doi:10.1016/j.idm.2020.03.001

14. X.Yu,Updated estimating infected population of Wuhan coronavirus in different poicyscenariousibySIRmodel,2020.http://unigoettinggen.de/en/infectious+diseases/619691.html.

15. ChenXidai, JingYang, KaifaWang, Evaluation of Prevention and control interventions and its impact on the epidemic of coronavirus disease 2019 in Chongqing and Guizhou Provinces, Mathematical Bioscience and engineering,2020,17,pp.27812791. idoi:i10.3934/mbe.2020152

16. Zhihua Bai,Yuegong, Xiaodongtian, YingCao, WenjunLiu, Jingli, The Rapid Assesment and Early Warning Models for COVID-19,National Library of Medicine,PubMedA,SpringerLink,2020.

17. F. Benjamin Maier, Drick Brockmann, Effective containment explains sub exponential growth in recent confirmed COVID-19 cases in China, Science, 2020, DOI:10.1126/science.abb4557

18. Steven Sanche,Yen Ting Lin, ChonggangXu, Ethan Romero-Severeson, NickHengartner, RuianKetHigh Contagiousness and rapid spread of severe Acute Respiratory Syndrome Coronavirus 2,Emerging InfectiousDiseases, 2020,26

19. Gaur, Naveen, Ram Niwash Mahia, and Om Prakash Mahela. "A Novel Method for Detection of Electric Transmission Line Faults Using Discrete Wavelet Transform."International Journal of Electrical and Electronics Engineering Research (IJEEER) 4. 5, O ct 2014, 63-74

20. David Smith, Lang Moore, The SIR Model for spread disease-The differential Equation Model Mathematical Association of America,2004

\section{URL Link}

21. https://docs.google.com/spreadsheets/d/17oWtuy98FkRCYNnBHGyPNEHPfx

VQP13M2z3kiSKRm0/edit?usp=sharing

\section{BIBLOGRAPHY}

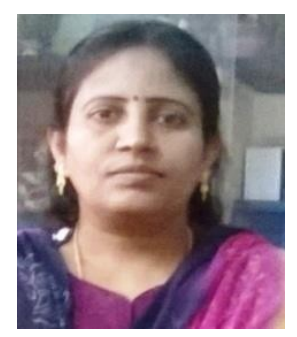

Dr. B. Vennila is from Perungalathur near Chennai. She is working as an Associate professor in SRM Institute of science and technology, Chennai. She received her Ph.D. degree in the area of Fluid dynamics from SRM University Chennai in 2010.She completed her MPhil mathematics from Alagappa University, Karaikudi in the year 1996. B.sc and M.sc Mathematics from Seethalakshmi Achi College for women, Karaikudi in the year 1990-1995. Her area of research interest is rotating fluid and stretching sheet problems. Sheisa member of ISTE, IMS, IAENG and ISCO. 


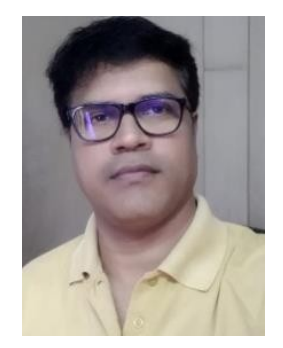

Mr. J. Sasikumar is from Chennai, India. He is working as Assistant Professor, Department of Mathematics in SRM Institute of Science and Technology, Kattankulathur-603203.He submitted his Ph.D. thesis to SRM Institute of Science and Technology in September 2019, doing research in the area fluid dynamics. He completed M.Phil. (Mathematics) with specialization in Number Theory and M.Sc. (Mathematics) during the year 1987-1991 from R.K.M. Vivekananda College, Chennai. He has total of 28 years of teaching experience in science and engineering colleges. He is the member of ISTE and IMS. 